\title{
Seeing Race: N170 Responses to Race and Their Relation to Automatic Racial Attitudes and Controlled Processing
}

\author{
Renana H. Ofan, Nava Rubin, and David M. Amodio
}

\begin{abstract}
We examined the relation between neural activity reflecting early face perception processes and automatic and controlled responses to race. Participants completed a sequential evaluative priming task, in which two-tone images of Black faces, White faces, and cars appeared as primes, followed by target words categorized as pleasant or unpleasant, while encephalography was recorded. Half of these participants were alerted that the task assessed racial prejudice and could reveal their personal bias ("alerted" condition). To assess face perception processes, the
\end{abstract}

\section{INTRODUCTION}

Human social interactions often begin with the perception of a face-a process accomplished in as little as $170 \mathrm{msec}$ (Bentin, Allison, Puce, Perez, \& McCarthy, 1996). Yet the way we perceive a face within a social interaction may be influenced by prior knowledge about social categories and the motivations we bring into the situation (Eberhardt, Dasgupta, \& Banaszynski, 2003). In the present research, we examined how previously learned associations with race may influence the earliest stages of visual face processing and how these effects may have implications for the control of behavioral responses.

\section{Perceiving Faces}

The initial perception of a face entails a rapid and concerted set of processes. One of the earliest processes involves the structural encoding of face components into a cohesive and meaningful object (i.e., a face). This encoding process has been associated with a component of the ERP called the N170, a negative polarity neuroelectric signal that occurs just $170 \mathrm{msec}$ after the presentation of a face and is most pronounced at right occipito-temporal scalp sites (Bentin et al., 1996). This N170 component reflects activity in multiple occipito-temporal structures linked to face processing (Deffke et al., 2007), including the fusiform and other temporal regions (Herrmann, Ehlis, Muehlberger, \& Fallgatter, 2005), consistent with fMRI studies indicating the involvement of the fusiform gyrus in face processing (e.g., Haxby, Hoffman, \& Gobbini, 2000; Puce,

New York University
N170 component of the ERP was examined. For all participants, stronger automatic pro-White bias was associated with larger N170 amplitudes to Black than White faces. For participants in the alerted condition only, larger N170 amplitudes to Black versus White faces were also associated with less controlled processing on the word categorization task. These findings suggest that preexisting racial attitudes affect early face processing and that situational factors moderate the link between early face processing and behavior.

Allison, Gore, \& McCarthy, 1995). These studies reveal that faces are processed very quickly and at relatively early stages in the hierarchy of visual processing.

Although the N170 is consistently responsive to faces, the psychological significance of this effect remains a matter of some debate. For example, the N170 amplitude is larger in response to clear upright faces of conspecifics than to nonface stimuli, suggesting that it reflects a facespecific response. Interestingly, when comparing responses to different types of faces, the N170 is often larger in response to inverted faces and faces of close heterospecifics than to upright human faces (de Haan, Pascalis, \& Johnson, 2002; Rossion et al., 2000). In this case, an inverted face constitutes a deviation from typical faces, in the sense that it contains the same components but in a different orientation than is normally encountered. Along these lines, Halit, de Haan, and Johnson (2000) found that atypical and unattractive faces elicit larger N170 amplitudes, compared with normative faces, in a passive viewing task. ${ }^{1}$ These findings suggest that, when comparing N170 amplitudes associated with different faces, a larger relative N170 amplitude may reflect additional processing involved in the perception of a face that deviates from one's normative representation of a face.

\section{Influences of Race on Face Perception}

Behavioral studies of face perception have shown that race is identified rapidly (Blair, Judd, \& Fallman, 2004), suggesting that racial information may influence early stages of visual processing. Race effects on face perception have also been examined by measuring the N170 component 
while participants viewed Black and White American faces. Interestingly, findings in this literature have been mixed with some studies observing no differences (e.g., He, Johnson, Dovidio, \& McCarthy, 2009; Wiese, Stahl, \& Schweinberger, 2009; Caldara, Rossion, Bovet, \& Hauert, 2004; Caldara et al., 2003), some observing larger N170 effects for the ingroup (Ito \& Urland, 2005), and others observing larger effects for the outgroup (Walker, Silvert, Hewstone, \& Nobre, 2008). These inconsistencies are likely because of differences in experimental tasks used in these studies vis-à-vis the expectancies and the response strategies adopted by participants during task completion. For example, tasks that involve the categorization of race (or gender) may focus participants on categorical differences (e.g., Ito \& Urland, 2005), whereas memory tasks (e.g., the $n$-back) do not (Walker et al., 2008), and for this reason patterns of perceptual and attentional processing are likely to differ. In addition, few prior N170 studies of race perception have controlled for low-level visual factors (e.g., luminance and contrast) in their stimuli, and therefore, it is unclear whether previously observed race effects were because of participants' cognitive representations of race or to these extraneous visual factors. Thus, although racial information appears to affect early face perception, important questions about the visual properties involved in race perception remain.

The effect of race on early face processing may have important implications for social behavior. Some research has asked whether early attentional effects of race relate to participants' implicit racial preferences and subsequent behavior. For example, in a study using the shooter task, in which outgroup members may be perceived as threatening, Correll, Urland, and Ito (2006) found that the P2 ERP component, peaking $\sim 180 \mathrm{msec}$ and associated with early attentional processes, was larger to outgroup than ingroup faces and that a larger race effect was associated with a pattern of stronger racial bias in behavior. Using a different task, in which outgroup faces provided cues to engage control, Amodio (2010) found larger P2 component amplitudes to outgroup versus ingroup faces. But in this task, in which Black faces provided a cue for control, larger P2 amplitudes to Black than White faces predicted better behavioral control on a stereotyping task among low-prejudice participants. These studies demonstrate that early attentional responses to ingroup versus outgroup faces may relate to racial biases and the goal-directed regulation of behavior. However, to date, research has not examined the critical relationship between visual face perception processes and behavioral patterns of automatic or controlled responses to race.

\section{The Present Study}

The goal of the present study was to examine the influence of race on early visual face processing, as indicated by the N170 component of the ERP, using White (e.g., Caucasian) and Black (e.g., African American) face stimuli that con- trolled for potentially confounding visual properties (i.e., luminance and contrast). A second goal was to examine the relationship between N170 differences to Black versus White faces and automatic and controlled forms of behavior among subjects in our primarily White sample. In particular, we asked whether automatic racial preferences were associated with differences in the N170 response to Black versus White faces. We also examined the effects of race perception on controlled processing. Past research has shown that participants' goals in a task can modulate whether attention to race impairs (Amodio, 2009; Lambert et al., 2003) or promotes (Amodio, 2010; Mendoza, Gollwitzer, \& Amodio, 2010; Monteith, 1993) response control. In this study, we manipulated participants' concern about responding with racial prejudice during the task by alerting some participants to the fact that the task assessed racial associations (although all responses were made privately). This alerting information was intended to raise subjects' concerns about failing to meet their personal standards of responding without prejudice, which has been shown to elicit vigilance to racial cues (Monteith, 1993). On the basis of prior research, we expected that this "alerting" manipulation would affect the link between N170 responses to race and controlled responding on the task (Amodio, 2009; Lambert et al., 2003).

\section{Experimental Approach}

In the present study, White and Black faces were presented as primes in a sequential evaluative priming task that assessed evaluative associations with race (Fazio, Jackson, Dunton, \& Williams, 1995). As in past N170 studies (e.g., Carmel \& Bentin, 2002), images of cars were also included as nonface primes. On each trial, the prime stimulus was followed by a positive or negative adjective, which participants quickly categorized as either "pleasant" or "unpleasant." White American participants tend to show a pattern of bias on this task, such that White faces facilitate the categorization of pleasant words more than unpleasant words and, to a lesser extent, Black faces facilitate the categorization of unpleasant words more than pleasant words (e.g., Devine, Plant, Amodio, Harmon-Jones, \& Vance, 2002; Fazio et al., 1995). This task is well designed for examining automatic and controlled processing because the trials are arranged in such a manner that automatic and controlled processes work in concert on some trials (e.g., Black-unpleasant trials) and in opposition on other trials (e.g., Black-pleasant trials).

When studying race perception, it is critical to control for extraneous stimulus effects on the perception of race in Black versus White individuals. To this end, images were rendered with black pixels only, on a white background ("two tone"; see Figure 1A), with the same ratio of black pixels to white pixels for all stimuli. After this transformation, all face and car stimuli were equal in luminance and contrast. To ensure that the race of these two-tone faces was highly discriminable at short presentation times, we conducted an independent pretest. This pretest indicated 
Figure 1. Sample stimuli (A) and task schematic (B).

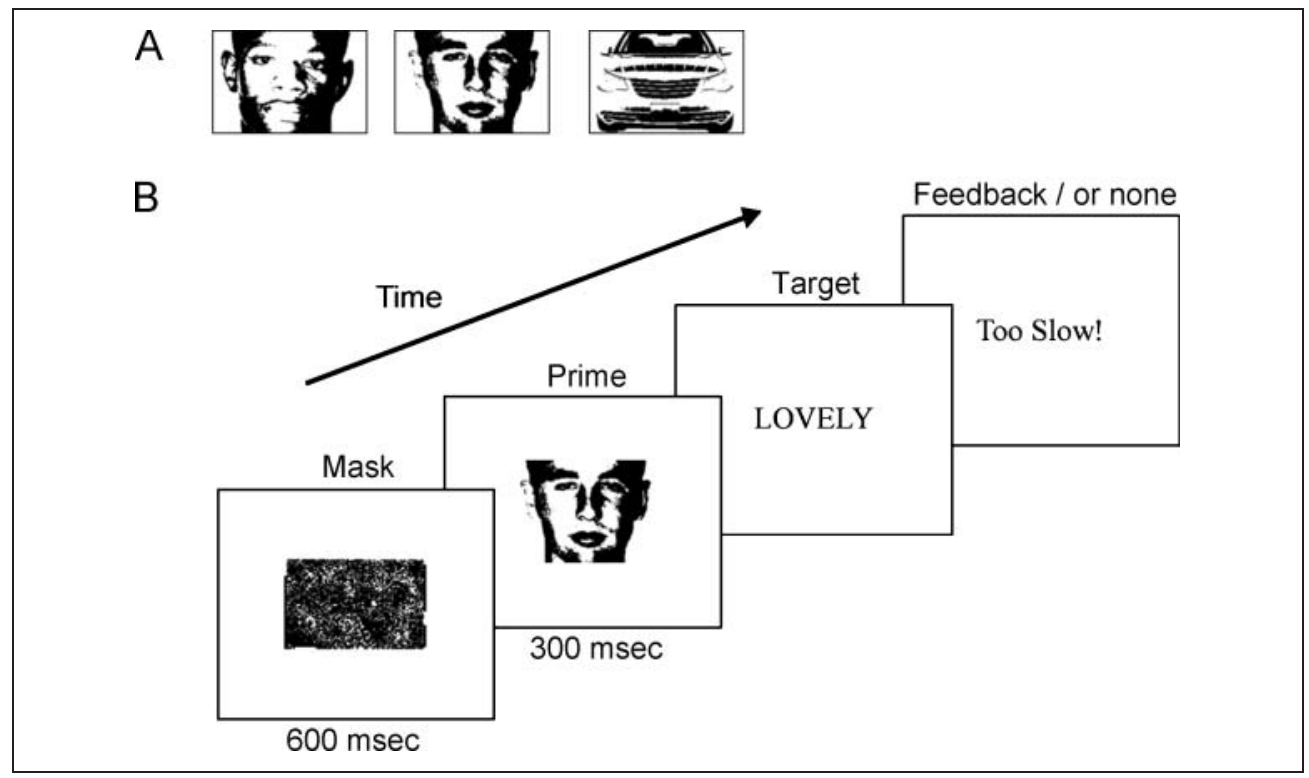

that the race of the two-toned faces was highly discriminable at presentation durations as short as $60 \mathrm{msec}$, and thus, two-toned images are effective at representing the racial identity of a face in the context of an N170 experiment (Ofan, Amodio, \& Rubin, 2011).

To examine N170 effects on automatic and controlled aspects of behavior, we modeled our data using Jacoby's (1991) process dissociation (PD) procedure (as in Amodio et al., 2004; Payne, 2001). This procedure is designed to identify separate and stochastically independent response patterns indicative of automatic and controlled processing. The control estimate represents the probability that one will respond in an accurate, goal-consistent manner irrespective of the race of the prime. By contrast, the automatic estimate represents the extent to which, when an error is made, that error reflects a race-biased association (see formulas in Methods).

\section{Hypotheses}

We tested two main hypotheses. The first hypothesis was that automatic racial attitudes, as indicated by task behavior, would be associated with differences in N170 amplitudes to Black versus White faces regardless of the condition. Previous research has shown that implicit Black-White racial bias is typically driven by ingroup positivity effects rather than by outgroup negativity (Conrey, Sherman, Gawronski, Hugenberg, \& Groom, 2005; Dovidio, Kawakami, Johnson, Johnson, \& Howard, 1997; Fazio et al., 1995), and thus, we expected that any racial differences in N170 responses would be associated with participants' degree of automatic pro-White bias. Therefore, in line with evidence for larger N170 responses to atypical and unattractive faces, we expected that stronger automatic proWhite associations would be associated with larger N170 responses to Black than White faces.
The second hypothesis concerned the relationship between the N170 effect and response control on the task, as a function of condition. We hypothesized that the alerting manipulation would increase participants' vigilance toward outgroup faces and that the increased vigilance to the face primes would interfere with their ability to respond to the main task of categorizing target words. Thus, we expected that, in the alerted condition, larger N170 amplitudes to Black (versus White) faces would be associated with lower estimates of control on the categorization task.

\section{METHODS}

\section{Participants}

Thirty-four right-handed (21 women and 13 men), Americanborn, native English-speaking Introductory Psychology students (82\% White and 18\% Asian) participated individually for extra course credit. Participants were randomly assigned to the alerted or control condition.

\section{Procedure}

After providing informed consent, participants were prepared for EEG recording and received task instructions. Participants were told that, on each trial, they would see a picture followed by a word, and they should categorize the word as pleasant or unpleasant via button press. Participants assigned to the "alerted" condition were additionally informed that the task was designed to examine racial prejudice and that their responses on certain trials could reveal an influence of racial bias (e.g., when erroneously categorizing a pleasant word as "unpleasant" following a Black face). In both conditions, participants were told that their responses would be made privately and confidentially. 


\section{Materials}

Prime stimuli included two-tone images of 10 Black male faces, 10 White male faces, and 10 cars (front view). All images were $250 \times 165$ pixels $\left(1 \times 0.66^{\circ}\right.$ visual angle $)$, with equal proportions of black pixels and white pixels and, therefore, equalized for luminance and contrast. Target words included 10 pleasant and 10 unpleasant words that were semantically unrelated to Black or White stereotypes or to cars (e.g., laughter, beauty, pain, and disgusting). All stimuli were presented in the center of the computer screen.

The task included 20 practice trials and 240 experimental trials. Trials representing the six possible types of prime-target combinations were presented in random order and were equally probable. Trials began with a pattern mask that served as a fixation mark $(600 \mathrm{msec})$, followed by a prime image $(300 \mathrm{msec})$ and then a target word that remained onscreen until a response was made. Participants were encouraged to respond within $600 \mathrm{msec}$, but responses were recorded until 1500 msec. A "too slow!" message followed responses that exceeded $600 \mathrm{msec}$. This deadline was used to elicit a sufficient distribution of errors for analysis. Participants received accuracy feedback on practice but not experimental trials. Stimuli and recording triggers were presented using DMDX software (Forster \& Forster, 2003).

\section{Behavioral Data Processing}

Responses with latencies between 200 and $1000 \mathrm{msec}$ were included in analyses, thereby excluding responses that likely associated with action slips or inattention (0.3\% of trials). Response latency scores for correct responses were submitted to natural log transformation and averaged as a function of trial type. Accuracy scores were computed as a function of trial type.

To derive independent estimates of automatic and controlled response patterns, formulas based on the PD procedure were used (Payne, 2001). For each prime type, the control estimate was quantified as $P$ (correct response on congruent trials) $-P$ (incorrect response on incongruent trials), where congruent refers to prejudice-consistent trials (e.g., Black-unpleasant trials) and incongruent refers to prejudice-inconsistent trials (e.g., Black-pleasant trials). This index is equivalent to general response accuracy irrespective of race, rescaled for the PD framework to range from -1 to 1 . In other words, the control estimate represents one's success in responding accurately to the target, regardless of the race of the face prime. The automatic estimate reflects the tendency to erroneously associate Black faces with negative words [i.e., Black-pleasant errors / $(1-$ control $)]$. PD automatic estimates were also computed for White face and car trials but scored to reflect a positivity bias [e.g., White-unpleasant errors / $(1-$ control $)] .{ }^{2}$ This method for examining patterns of automatic and controlled processes in behavior has been used successfully in several previous studies (e.g., Amodio, 2010; Huntsinger, Sinclair,
\& Clore, 2009; Stewart, von Hippel, \& Radvansky, 2009; Payne, 2001).

\section{EEG Recording and Processing}

EEG was recorded from $11 \mathrm{Ag} / \mathrm{AgCl}$ electrodes (five midline sites: Fz, Fcz, Cz, Pz, Oz; six lateral-occipital sites: P7/8, $\mathrm{PO} 7 / 8, \mathrm{PO} / 10$ ), embedded in a stretch lycra cap (Electrode Arrays, El Paso, TX), positioned according to the 10-10 system, referenced on-line to the left earlobe $(\mathrm{k} \Omega<5)$, with a forehead ground. Vertical and horizontal eye movements were recorded to facilitate artifact scoring. Signals were amplified using a Neuroscan Synamps2 (El Paso, TX) with AC coupling, 15,100 Hz bandpass-filtered, and digitized at $1000 \mathrm{~Hz}$. Off-line, EEG was rereferenced to average earlobes, scored for movement artifact (4\% of the trials were rejected due to artifact), submitted to a regression-based eye blink correction procedure, and then digitally filtered through a $1-15 \mathrm{~Hz}$ bandpass. To compute ERP waveforms, a 900-msec stimulus-locked epoch was selected for each artifact-free trial beginning $100 \mathrm{msec}$ before prime onset. Baseline correction procedures subtracted the average voltage during a 100-msec prestimulus period from each epoch, and then epochs were averaged within their respective trial types. For each subject, the N170 was scored as the peak negative amplitude between 150 and $210 \mathrm{msec}$ following face onset at lateral-occipital sites.

Two participants were excluded from analysis-one for extreme outlying $(>3 S D)$ response latency scores and one because of excessive EEG artifact-leaving 32 participants for analysis.

\section{RESULTS}

\section{Task Behavior}

Preliminary analyses examined accuracy rates and response latencies on the behavioral task. We expected that pleasant word categorizations would be facilitated by White faces relative to Black faces, whereas unpleasant words would be facilitated by Black faces relative to White faces (as in Fazio et al., 1995). Log-transformed response latencies were submitted to a 3 (Prime: Black face, White face, car) $\times 2$ (Target: pleasant, unpleasant) $\times 2$ (Condition: control, alerted) mixed-factorial ANOVA. The expected Prime $\times$ Target interaction was significant, $F(2$, $60)=6.84, p<.01$. Simple effects tests revealed that unpleasant words were categorized faster following Black faces than either White faces or cars, $p s<.05$. Responses to pleasant words were faster following White faces than following either Black faces $(p=.08)$ or cars $(p=.07)$, as expected, although these effects were marginal. The three-way interaction, which was not predicted, was not significant, $F(2,60)=0.95, p=.39$. Additionally, a main effect for condition, $F(1,30)=10.97, p<.01$, revealed slower responses in the alerted condition than the control 


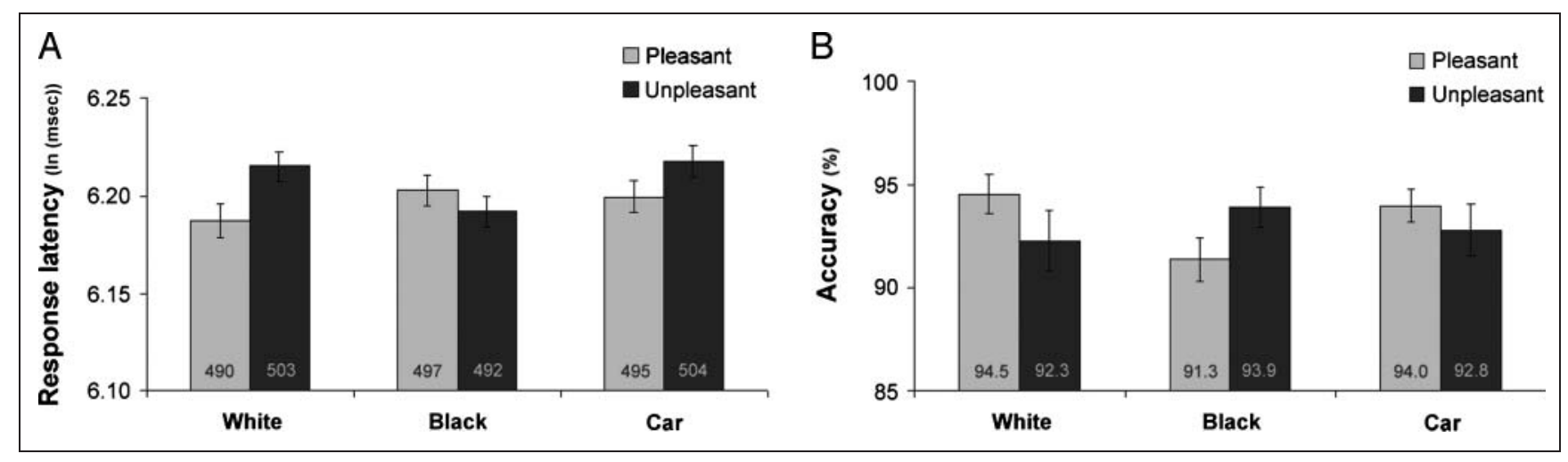

Figure 2. Response latencies in log millisecond with raw latencies labeled on each bar (A) and accuracy (B) as a function of trial type.

condition, consistent with the idea that these participants were concerned about responding with bias.

Overall performance accuracy ranged from $71 \%$ to $100 \%$ $(M=.93, S D=.06$, skewness $=-1.19)$, providing ample variability for the analysis accuracy rates and process dissociation estimates. Accuracy scores were submitted to a 3 (Prime) $\times 2$ (Target) $\times 2$ (Condition) mixed-factorial ANOVA. This analysis produced a marginally significant Prime $\times$ Target interaction, $F(2,60)=2.77, p=.07$ (see Figure 2B). Pairwise $t$ tests indicated greater accuracy for pleasant words following White faces and cars compared with Black faces, $p s<.05$. By contrast, accuracy for negative words was greater following Black faces compared with White faces or cars, $p s<.01$. Overall, task behavior indicated a pattern of racial bias driven by strong positive associations with the White ingroup, as in past work (e.g., Conrey et al., 2005; Fazio et al., 1995).

Mean process dissociation estimates of automaticity for White faces $(M=.48, S D=.34)$ and Black faces $(M=.58$, $S D=.31$ ) did not differ, $t(31)=-1.28, p=.21$, consistent with the idea that evaluative associations exist for both White and Black faces. These estimates were not significantly correlated, $r=.14, p=.45$, such that automatic evaluative responses to Black and White faces were largely independent. By contrast, process dissociation estimates of control for trials with White faces $(M=.87, S D=.11)$ and Black faces $(M=.85, S D=.09)$ were highly correlated, $r=$ $.76, p<.001$, consistent with the idea that the PD control estimate reflects general response control and overall task accuracy irrespective of race (e.g., Payne, 2005; Amodio et al., 2004). These control estimates did not differ statistically, $t(31)=-1.27, p=.21$, and they were uncorrelated with the automatic estimates, $p s>.11$. As in previous work, these collinear estimates of control were averaged to form a single index representing the ability to respond according to task goals without being influenced by race.

\section{ERP Effects}

\section{N170 Amplitude}

In a preliminary analysis to examine laterality effects, the mean N170 amplitude scores at the three lateral-occipital sites within each hemisphere were submitted to a preliminary 2 (Hemisphere: right vs. left) $\times 2$ (Condition: control vs. alerted) mixed-factorial ANOVA. This analysis produced a significant main effect for hemisphere, $F(1$, $30)=23.19, p<.001$, indicating that the N170 amplitudes were larger at the right occipito-temporal sites (right hemisphere: $M=-8.72, S D=4.94$; left hemisphere: $M=$ $-6.38, S D=4.11$ ), as found in past research (e.g., Bentin et al., 1996). Subsequent analyses focused on right hemisphere N170 amplitudes scored at the PO10 electrode site at which the N170 was maximal (Figure 3).

To examine the effect of race on N170 amplitudes, amplitudes were submitted to a 3 (Prime: White face vs. Black face vs. car) $\times 2$ (Condition: control vs. alerted) withinsubjects ANOVA. This analysis produced a main effect for prime, $F(2,60)=9.77, p<.01$. Simple effect analyses indicated that N170 amplitudes were larger for both White faces, $t(31)=-2.93, p<.01$, and Black faces, $t(31)=$ $-3.58, p<.01$, compared with cars, replicating past findings (Carmel \& Bentin, 2002). However, N170 responses to Black and White faces did not differ significantly,

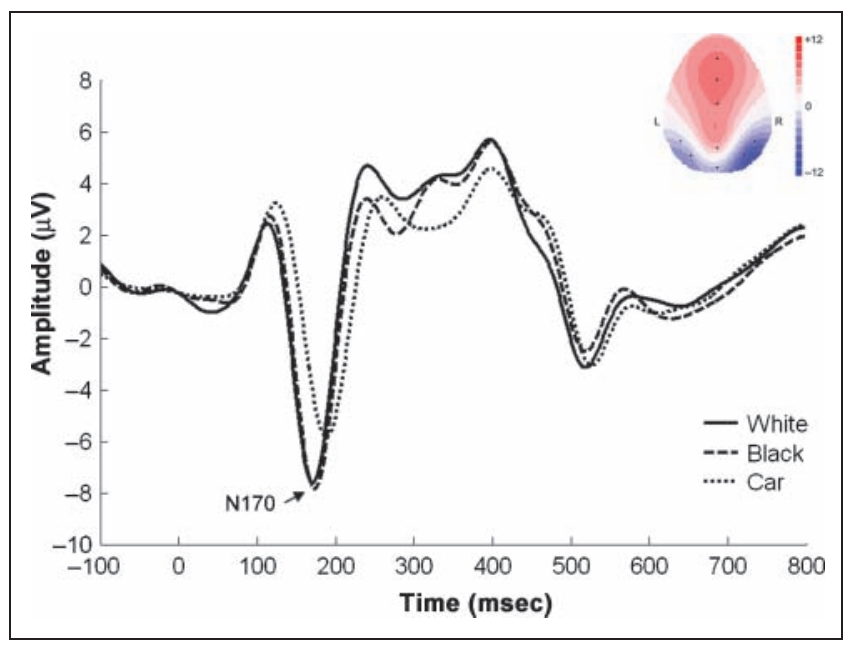

Figure 3. ERP waveforms recorded at PO10 associated with each prime stimulus. The zero millisecond point represents the onset of the prime on the screen. Top right inset: Scalp topographic voltage map at the peak of the N170 component. 
$t(31)=1.19, p=.24$, consistent with previous null findings (Caldara et al., 2003, 2004; see also Walker et al., 2008; Ito \& Urland, 2005). Effects for neither condition nor interaction were significant, $F$ S $<1$.

\section{N170 Latency}

A 3 (Prime) $\times 2$ (Condition) within-subjects ANOVA on N170 peak latency produced only an effect for prime, $F(2,60)=94.76, p<.001$. N170 latencies were significantly longer for cars $(M=191, S D=14)$ than to either White faces $(M=173, S D=11)$ or Black faces $(M=176$, $S D=11), p s<.001$, closely replicating previous findings (e.g., Carmel \& Bentin, 2002). Furthermore, N170 latencies were significantly longer for Black faces than White faces, $t(31)=-4.21, p<.001$, as in Wiese et al. (2009), suggesting slower processing of Black faces relative to White faces.

\section{Relationship between N170 Amplitude and Behavior}

Our main hypotheses concerned the relationship between N170 amplitudes to Black versus White faces and behavior. Although the average N170 amplitudes to Black and White faces did not differ, there was substantial variability, with some subjects showing greater amplitude in response to Black than White faces and others showing the reverse. We therefore performed a set of regression analyses to examine the relation between individual differences in N170 amplitudes to Black versus White faces and behavioral measures of automatic and controlled response patterns. The Black versus White N170 difference scores were computed for each participant, with more negative values indicating larger N170 amplitudes to Black faces than White faces. These scores were mean-centered for testing interaction effects. Condition was coded as -1 (control) and 1 (alerted). The regression model included effects for the N170 difference score, condition, and their interaction as predictors, with process dissociation estimates of automaticity (for White and Black faces and cars separately) and control as outcome variables.

First, we tested our hypotheses regarding automatic racial bias. A regression analysis of PD automatic estimates for White faces produced a significant main effect for N170 amplitude difference, $\beta=-0.47, p<.01$. This effect indicated that, across conditions, stronger automatic White-positive associations were associated with larger N170 responses to Black versus White faces. This regression did not produce significant effects for condition (alerted vs. control) or the interaction. In separate analyses, this regression model did not reveal any significant effects for automatic estimates associated with Black faces $(p s>.29)$ or cars $(p s>.37)$. This pattern of results is consistent with research demonstrating the primacy of ingroup favoritism over outgroup negativity in automatic racial bias (Fazio et al., 1995; Brewer, 1991). More impor-

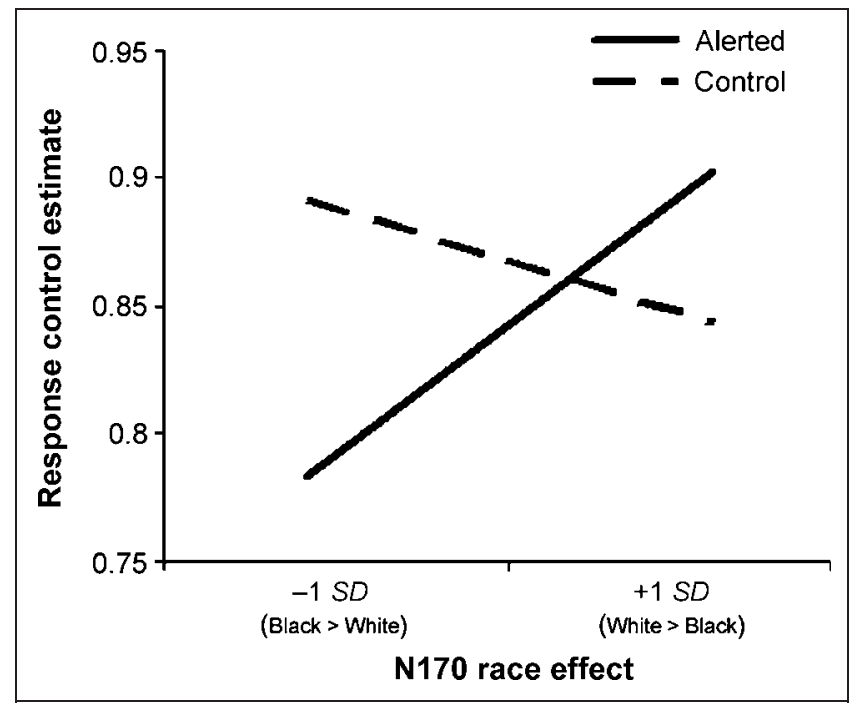

Figure 4. Predicted values of response control estimates illustrating the interaction of N170 race effect (N170 amplitude for Black faces minus the amplitude for White faces) and condition, computed at $1 S D$ below and above each mean.

tantly, this result revealed a relationship between the strength of participants' automatic racial attitudes and early-stage processing of Black versus White faces, as indicated by the N170.

Next, we examined the relationship between race perception and response control. The regression analysis of the average control estimate indicated only a significant interaction, $\beta=0.44, p=.01$. Simple slope analyses indicated that in the control condition, there was no significant relationship between the $\mathrm{N} 170$ difference and the estimate of response control, $\beta=-0.30, p=.22$. However, in the alerted condition, larger N170 difference scores were associated with higher response control estimates, $\beta=0.59$, $p=.02$ (Figure 4). That is, when alerted to the potential for bias, participants with larger N170 amplitudes to Black (vs. White) faces exhibited worse response control. This finding is consistent with the idea that, when participants in the alerted condition encounter a racial cue (Black face), enhanced processing of this cue interfered with responding on the main word categorization task.

\section{DISCUSSION}

Social interactions often begin with the perception of a face, yet even the earliest face perception process can be influenced by a person's preexisting attitudes and beliefs. Indeed, we found that preexisting automatic preferences for participants' White racial ingroup was associated with the relative degree of early processing of White and Black faces, as indicated by the N170 component of the ERP. Specifically, those with stronger automatic preferences for White people showed relatively larger N170 responses to Black than White faces. This finding is consistent with 
our hypothesis that people with stronger ingroup preferences may see outgroup faces as less normative and, thus, require greater engagement of early facial encoding processes. This interpretation is consistent with past research demonstrating enhanced N170 amplitudes in response to passive or incidental viewing of atypical faces (Halit et al., 2000; but see Freeman, Ambady, \& Holcomb, 2010, for effects related to task-relevant face processing). This relationship between pro-White bias and N170 amplitudes was evident across the experimental conditions, consistent with the idea that automatic racial attitudes can operate implicitly and independently of individuals' efforts to respond in a controlled manner.

Early processing of ingroup and outgroup faces may also influence subsequent behavioral responses, as a function of one's situational goals. For example, in real-life interracial interactions, majority group members are often concerned about appearing prejudiced and devote considerable effort to avoiding this appearance (Amodio, Kubota, Harmon-Jones, \& Devine, 2006). In the present experiment, we found that when participants were alerted to the possibility that responses to Black faces could reveal their racial preferences, stronger N170 responses to Black (vs. White) faces were also associated with worse response control. An interpretation of this finding is that, when the need for additional face processing resources for outgroup faces is combined with concern about showing prejudice toward outgroup faces, participants may require more time to respond accurately and, thus, may be more prone to control failures. In other words, the increased vigilance to face primes that "alerted" participants believed could reveal racial bias interfered with the ability to respond effectively on the main task of categorizing target words. This result suggests an interesting interaction between perceptual and social factors in the regulation of intergroup responses.

In addition to testing our main hypotheses, the present work includes some notable advances in the study of early race perception. First, by using two-tone face stimuli, which equalized the luminance and contrast of Black and White faces, we controlled for extraneous factors that may have influenced findings in previous studies. Second, by presenting faces within the context of a task that produced meaningful behavioral indices of automatic and controlled processes, we were able to examine the relationship between early race perception and behavior-based ingroup favoritism and response control. Third, the inclusion of a visual control stimulus (the car prime) is common in the visual perception literature but relatively novel for social cognition studies. The inclusion of the car prime clarified that the N170 was indeed sensitive to faces. Furthermore, although both White faces and cars were evaluated more positively than Black faces on the task, the N170 effect was only associated with positivity toward White faces, indicating that the N170 effects were specific to intergroup social evaluation and not to general evaluative tendencies.

\section{The N170 and Race}

The present research focused on the relationship between the early processing of a face's race and different aspects of behavior and, thus, our analyses concerned individual differences. However, we did not observe an average difference for race across participants. Why? It appears that some participants exhibited larger N170s to Black faces, others to White faces, and others showed no difference. Indeed, several previous studies did not find N170 differences for race (e.g., Wiese et al., 2009). Interestingly, studies that found a race difference (in either direction) did not include nonface control stimuli. It is possible that the inclusion of only White and Black faces in these studies may have drawn more attention to the racial distinction. Additionally, the face stimuli in those previous studies were not equalized for luminance and contrast, and therefore, one cannot rule out that some (or all) of the previously observed race differences in the N170 were because of differences in those basic visual attributes associated with Black versus White faces rather than racial associations per se. Using two-tone stimuli, we also found that the N170 latency was slightly longer for Black than White faces, replicating Wiese et al. (2009). This may indicate the delayed processing of Black versus White faces at early stages of face perception. Although the interpretation is speculative, the effect likely represents the top-down influence of racial associations rather than bottom-up stimulus effects.

\section{Implications for Intergroup Social Cognition}

Our findings suggest that preexisting automatic racial attitudes may influence the early processing of a face, within 170 msec of encountering an individual. Although much previous research has shown that such attitudes influence a broad range of sociocognitive processes, such as biased social perceptions, information processing, and judgments (Fiske, 1998), the present research suggests that such attitudes may also affect the encoding of ingroup and outgroup faces in vision. This finding extends the range of processes influenced by implicit racial attitudes and, in doing so, broadens the set of mechanisms through which racial attitudes can introduce bias in an intergroup social interaction. Researchers interested in interventions to reduce the effects of implicit bias in behavior may wish to consider the role of early visual processing and, as such, develop interventions that address potential differences in these early face perception processes.

Our research also sheds new light on the role of race in cueing plans for response control. Much previous research has shown that, when alerted to the possibility of showing prejudice, perceivers with egalitarian beliefs will be vigilant for racial cues and, when a cue is encountered, engage in a more careful and controlled pattern of response (Amodio, 2010; Mendoza et al., 2010; Monteith, 1993). In the present study, as in past work, the presentation of an outgroup 
(Black) face appeared to have served as such a cue. Whereas Amodio (2010) showed that rapid attentional processing of a face was related to cue detection and control, the present findings suggest that vigilance for racial cues is influenced by a perceiver's visual processing of the face. However, in our experimental task, efforts allocated to responding without racial bias detracted from efforts to categorize the valence of target words. This resulted in responding with less control on the main task-a task that is irrelevant to racial concerns. We would predict that, if given the opportunity, these participants would have shown increased control on a task that directly assessed the regulation of prejudiced behavior. More broadly, these findings suggest that, for some individuals, vigilance for racial cues tunes the early processing of a face in a way that facilitates the engagement of goal-consistent behavior. As a whole, this research begins to explore the role of early perceptual processes in intergroup social cognition.

\section{Implications for Perception}

The present research provides a novel test of top-down influences on early visual processing (e.g., Bar, 2003; Miyashita \& Hayashi, 2000). The traditional view in the vision literature is that face perception, like most other object recognition processes, is achieved primarily through bottom-up processes of image and shape analysis (e.g., Ullman, 1996; Bruce \& Young, 1986). Recent reports of race effects on N170 amplitudes are, therefore, interesting, because they suggest a top-down influence of social cognition and group membership on what was previously considered a purely bottom-up process. By controlling for critical stimulus confounds, our data provide new, stronger evidence of meaningful top-down effects on early-stage face processes in visual perception. In summary, these findings advance our understanding of how perceptual processes contribute to the regulation of social behavior and add new evidence to classic debates on how topdown processes may influence perception.

Reprint requests should be sent to David M. Amodio, Department of Psychology, New York University, New York, NY 10003, or via e-mail: david.amodio@nyu.edu.

\section{Notes}

1. A larger N170 amplitude to nonnormative faces has been observed during tasks in which the viewing of a face is passive and/or incidental (e.g., de Haan et al., 2002; Halit et al., 2000; Rossion et al., 2000), as in the present experiment. However, this pattern may not apply when the main task goal is to categorize the identity of a face. For example, in a study by Freeman et al. (2010), the participants' task was to categorize faces according to gender. The male and female stimuli varied in gender typicality, and the authors observed larger N170 amplitudes to more typical faces. Thus, in Freeman et al. (2010), typicality was directly relevant to the main task of categorizing gender identity, and more typical faces engaged a stronger "match" effect. In the present study, faces were viewed incidentally, and therefore, larger N170 amplitudes were interpreted as reflected greater atypicality.

2. In cases where control was equal 1 and, therefore, PD automatic estimates were not defined, missing values were replaced with 0 , indicating no bias.

\section{REFERENCES}

Amodio, D. (2009). Intergroup anxiety effects on the control of racial stereotypes: A psychoneuroendocrine analysis. Journal of Experimental Social Psychology, 45, 60-67.

Amodio, D. (2010). Coordinated roles of motivation and perception in the regulation of intergroup responses: Frontal cortical asymmetry effects on the P2 event-related potential and behavior. Journal of Cognitive Neuroscience, 22, 2609-2617.

Amodio, D., Harmon-Jones, E., Devine, P., Curtin, J., Hartley, S., \& Covert, A. (2004). Neural signals for the detection of unintentional race bias. Psychological Science, 15, 88-93.

Amodio, D. M., Kubota, J. T., Harmon-Jones, E., \& Devine, P. G. (2006). Alternative mechanisms for regulating racial responses according to internal vs. external cues. Social Cognitive and Affective Neuroscience, 1, 26-36.

Bar, M. (2003). A cortical mechanism for triggering top-down facilitation in visual object recognition. Journal of Cognitive Neuroscience, 15, 600-609.

Bentin, S., Allison, T., Puce, A., Perez, E., \& McCarthy, G. (1996). Electrophysiological studies of face perception in humans. Journal of Cognitive Neuroscience, 8, 551-565.

Blair, I., Judd, C., \& Fallman, J. (2004). The automaticity of race and Afrocentric facial features in social judgments. Journal of Personality and Social Psychology, 87, 763-778.

Brewer, M. (1991). The social self: On being the same and different at the same time. Personality and Social Psychology Bulletin, 17, 475-482.

Bruce, V., \& Young, A. (1986). Understanding face recognition. British Journal of Psychology (London, England: 1953), 77, 305.

Caldara, R., Rossion, B., Bovet, P., \& Hauert, C. (2004). Event-related potentials and time course of the "other-race" face classification advantage. NeuroReport, 15, 905.

Caldara, R., Thut, G., Servoir, P., Michel, C., Bovet, P., \& Renault, B. (2003). Face versus non-face object perception and the "other-race" effect: A spatio-temporal event-related potential study. Clinical Neurophysiology, 114, 515-528.

Carmel, D., \& Bentin, S. (2002). Domain specificity versus expertise: Factors influencing distinct processing of faces Cognition, 83, 1-29.

Conrey, F. R., Sherman, J. W., Gawronski, B., Hugenberg, K., \& Groom, C. J. (2005). Separating multiple processes in implicit social cognition: The quad model of implicit task performance. Journal of Personality and Social Psychology, 89, 469-487.

Correll, J., Urland, G., \& Ito, T. (2006). Event-related potentials and the decision to shoot: The role of threat perception and cognitive control. Journal of Experimental Social Psychology, 42, 120-128.

de Haan, M., Pascalis, O., \& Johnson, M. J. (2002). Specialization of neural mechanisms underlying face recognition in human infants. Journal of Cognitive Neuroscience, 14, 99-209.

Deffke, I., Sander, T., Heidenreich, J., Sommer, W., Curio, G., Trahms, L., et al. (2007). MEG/EEG sources of the 170-ms response to faces are co-localized in the fusiform gyrus. Neuroimage, 35, 1495-1501.

Devine, P. G., Plant, E. A., Amodio, D. M., Harmon-Jones, E., \& Vance, S. L. (2002). The regulation of explicit and implicit race bias: The role of motivations to respond without 
prejudice. Journal of Personality and Social Psychology, 82, 835-848.

Dovidio, J., Kawakami, K., Johnson, C., Johnson, B., \& Howard, A. (1997). On the nature of prejudice: Automatic and controlled processes. Journal of Experimental Social Psychology, 33, 510-540.

Eberhardt, J. L., Dasgupta, N., \& Banaszynski, T. L. (2003). Believing is seeing: The effects of racial labels and implicit beliefs on face perception. Personality and Social Psychology Bulletin, 29, 360-370.

Fazio, R., Jackson, J., Dunton, B., \& Williams, C. (1995). Variability in automatic activation as an unobtrusive measure of racial attitudes: A bona fide pipeline? Journal of Personality and Social Psychology, 69, 1013-1027.

Fiske, S. T. (Ed.) (1998). Stereotyping, prejudice, and discrimination. New York: McGraw-Hill.

Forster, K., \& Forster, J. (2003). DMDX: A Windows display program with millisecond accuracy. Behavior Research Methods, Instruments, \& Computers, 35, 116-124.

Freeman, J., Ambady, N., \& Holcomb, P. (2010). The face-sensitive N170 encodes social category information. NeuroReport, 21, 24-28.

Halit, H., de Haan, M., \& Johnson, M. (2000). Modulation of event-related potentials by prototypical and atypical faces. NeuroReport, 9, 1871-1875.

Haxby, J., Hoffman, E., \& Gobbini, M. (2000). The distributed human neural system for face perception. Trends in Cognitive Sciences, 4, 223-232.

He, Y., Johnson, M., Dovidio, J., \& McCarthy, G. (2009). The relation between race-related implicit associations and scalp-recorded neural activity evoked by faces from different races. Social Neuroscience, 4, 426.

Herrmann, M., Ehlis, A., Muehlberger, A., \& Fallgatter, A. (2005). Source localization of early stages of face processing Brain Topography, 18, 77-85.

Huntsinger, J., Sinclair, S., \& Clore, G. (2009). Affective regulation of implicitly measured stereotypes and attitudes: Automatic and controlled processes. Journal of Experimental Social Psychology, 45, 560-566.

Ito, T., \& Urland, G. (2005). The influence of processing objectives on the perception of faces: An ERP study of race and gender perception. Cognitive, Affective, \& Behavioral Neuroscience, 5, 21-36.

Jacoby, L. (1991). A process dissociation framework: Separating automatic from intentional uses of memory. Journal of Memory and Language, 30, 513-541.

Lambert, A., Payne, B., Jacoby, L., Shaffer, L., Chasteen, A., \& Khan, S. (2003). Stereotypes as dominant responses: On the "social facilitation" of prejudice in anticipated public contexts. Journal of Personality and Social Psychology, 84, $277-295$.

Mendoza, S., Gollwitzer, P., \& Amodio, D. (2010). Reducing the expression of implicit race bias: Reflexive control through implementation intentions. Personality and Social Psychology Bulletin, 36, 512-523.

Miyashita, Y., \& Hayashi, T. (2000). Neural representation of visual objects: Encoding and top-down activation. Current Opinion in Neurobiology, 10, 187-194.

Monteith, M. (1993). Self-regulation of prejudiced responses: Implications for progress in prejudice-reduction efforts. Journal of Personality and Social Psychology, 65, 469-485.

Ofan, R., Amodio, D., \& Rubin, N. (2011). Top-down influences on race categorization. Unpublished manuscript.

Payne, B. (2001). Prejudice and perception: The role of automatic and controlled processes in misperceiving a weapon. Journal of Personality and Social Psychology, 81, 181-192.

Payne, B. K. (2005). Conceptualizing control in social cognition: How executive functioning modulates the expression of automatic stereotyping. Journal of Personality and Social Psychology, 89, 488-503.

Puce, A., Allison, T., Gore, J., \& McCarthy, G. (1995). Facesensitive regions in human extrastriate cortex studied by functional MRI. Journal of Neurophysiology, 74, 1192-1199.

Rossion, B., Gauthier, I., Tarr, M. J., Despland, P., Bruyer, R., Linotte, S., et al. (2000). The N170 occipito-temporal component is delayed and enhanced to inverted faces but not to inverted objects: An electrophysiological account of face-specific processes in the human brain. NeuroReport, 11, 69-74.

Stewart, B., von Hippel, W., \& Radvansky, G. (2009). Age, race, and implicit prejudice. Psychological Science, 20, 164.

Ullman, S. (1996). High-level vision. Cambridge, MA: MIT Press.

Walker, P., Silvert, L., Hewstone, M., \& Nobre, A. (2008). Social contact and other-race face processing in the human brain. Social Cognitive and Affective Neuroscience, 3, 16.

Wiese, H., Stahl, J., \& Schweinberger, S. (2009). Configural processing of other-race faces is delayed but not decreased. Biological Psychology, 81, 103-109. 